\title{
Comparing two different methods of identifying alcohol related problems in the emergency department: a real chance to intervene?
}

\author{
A Hadida, N Kapur, K Mackway-Jones, E Guthrie, F Creed
}

\begin{abstract}
Objectives-To examine the feasibility of screening for alcohol problems in a representative flow sample of patients attending a busy UK emergency department. To compare two methods of identifying alcohol related problems in the emergency department.

Methods-Brief interview administered by the same interviewer to a representative flow sample of 429 patients attending a single accident and emergency department over a six week period. Measures included a CAGE questionnaire and assessments by the patient and staff as to whether the attendance was alcohol related.

Results-413 patients (96\%) were successfully screened. Of these, $115(28 \%)$ patients were considered to have an alcohol related attendance on the basis of the CAGE questionnaire or the staff assessment. Head injuries and psychiatric presentations were particularly likely to be associated with alcohol misuse. Compared with those identified by staff, patients scoring above threshold on the CAGE were more likely to attend during routine working hours and recognise they had an alcohol problem.
\end{abstract}

Conclusions-Emergency departments may provide an opportunity for the early prevention of alcohol related difficulties. However, patients with alcohol problems who present to the emergency department are not a homogenous group. Different screening methods identify different groups of patients, who in turn may respond to different forms of intervention. Further research examining the efficacy and feasibility of different alcohol treatment approaches is needed to enable us to target specific interventions to those patients who might most benefit.

(Emerg Med f 2001;18:112-115)

Keywords: alcohol

Between $37 \%-50 \%{ }^{1-3}$ of all patients have consumed alcohol before presentation at emergency departments (EDs). Alcohol consumption has potentially serious consequences: it is an important contributing factor to various types of injury ${ }^{4-7}$ and those who present to EDs in an intoxicated state have a mortality rate of 2.4 times the normal population if they are followed up over a five year period. ${ }^{8}$ Despite the scale and seriousness of alcohol problems in ED patients, only a small percentage are identified as having alcohol problems by ED staff, and even fewer are referred to specialist alcohol services. $^{9}$

EDs may be the initial point of healthcare contact for patients with alcohol problems and could therefore provide an opportunity for the successful prevention of further alcohol related difficulties. A first step to providing services for this group of patients would be to identify those patients with alcohol problems who may be amenable to treatment. Studies conducted in EDs in London suggest that this may be a feasible option. ${ }^{10}{ }^{11}$ However, screening is not without its problems. Patients, especially those who are intoxicated, are highly unlikely to complete self report measures. ${ }^{10}$ Asking ED staff to complete questionnaires results in a higher pick up rate, but this still only identifies $5 \%$ of the expected number of patients with alcohol problems. ${ }^{10}{ }^{11}$ In the largest UK study of its type involving 17000 patients attending an ED in the north of England, the researchers were successfully able to screen less than one third of attendees. ${ }^{12}$ These figures perhaps reflect the extremely pressured work environment in many EDs.

In this study we wished to identify as accurately as possible the numbers of patients with alcohol related problems presenting to a busy ED by screening a representative "flow sample". We also wished to compare two methods of assessing alcohol related problems (the CAGE questionnaire ${ }^{13}$ and staff assessment) to ascertain whether they identified different groups of patients.

\section{Methods}

SUBJECTS

The study was carried out at Manchester Royal Infirmary over a six week period. The ED serves a deprived inner city area and sees approximately 67500 new patients per annum.

The day was divided into six time periods: 8 am-12 noon, 12 noon- 4 pm, 4 pm-8 pm, $8 \mathrm{pm}$-midnight, midnight $-4 \mathrm{am}$ and $4 \mathrm{am}-$ $8 \mathrm{am}$. Over the six week period subjects were recruited during each of the different time periods to avoid a sampling bias. Recruitment took place over a total of 336 hours (equivalent to 14 complete 24 hour periods). Every fifth consecutive patient aged 12 or over attending the ED during the specific time period was asked to complete a brief screening interview about alcohol consumption and problems (appendix). 
Table 1 Distribution of presenting complaint in relation to alcohol related attendance at the ED

\begin{tabular}{llcl}
\hline Presenting complaint & Total number & $\begin{array}{l}\text { Number (\%) } \\
\text { alcohol related }\end{array}$ & $\begin{array}{l}\text { Number (\%) } \\
\text { not alcohol related }\end{array}$ \\
\hline Head injury & 36 & $24(66)$ & $12(33)$ \\
Back pain & 14 & $2(14)$ & $12(86)$ \\
RTA & 30 & $4(13)$ & $26(87)$ \\
Hand injury & 46 & $3(6.5)$ & $43(93.5)$ \\
Abdominal pain & 33 & $6(18)$ & $27(82)$ \\
Limb injury & 70 & $13(19)$ & $57(81)$ \\
Chest pain & 29 & $0(0)$ & $29(100)$ \\
Respiratory & 25 & $1(4)$ & $24(96)$ \\
Psychiatric & 20 & $10(50)$ & $10(50)$ \\
Headache & 13 & $2(15)$ & $11(85)$ \\
Collapse & 26 & $4(15)$ & $22(84)$ \\
Assault & 22 & $10(45)$ & $3(10)$ \\
Gynaecology & 3 & $0(0)$ & \\
\hline
\end{tabular}

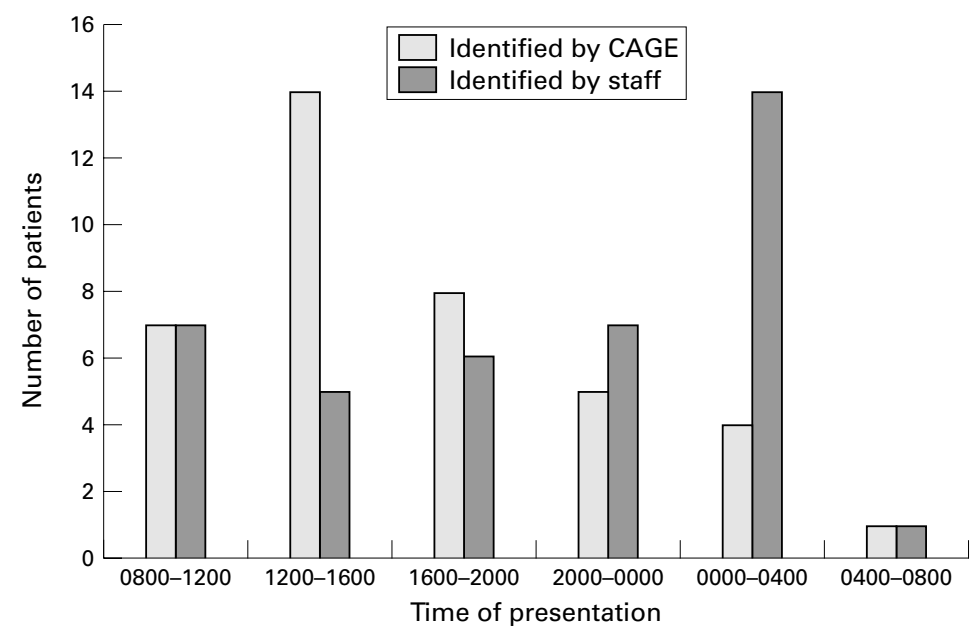

Figure 1 Time distribution of attendances at the ED related to alcohol problems.

Patients were approached after they had been triaged. The same interviewer, a fourth year medical student $(\mathrm{AH})$, interviewed them all. The interview was designed to be very brief so that any member of the ED department could administer it.

The patient's presenting complaint was noted and two alcohol related assessments were conducted.

(1) Staff assessment

After the patient had been examined and treated the ED doctor and the nurse who had seen the patient were asked to make a judgement as to whether the patient's presenting problem was alcohol related.

(2) Patient's assessment

Patients were asked whether they thought that their presenting problem was related to excessive alcohol consumption. They were then asked to complete the CAGE screening questionnaire. ${ }^{12}$ This contains four items, which relate to drinking habits. If the subject endorses two or more items it is probable that he or she is a problem drinker.

STATISTICAL ANALYSIS

Non-parametric analyses were carried out on a personal computer using SPSS (version 7)

Results

A total of 429 patients were approached during the study period. Three patients refused, 13 were too ill to be interviewed. Altogether 413 patients were therefore included in the study. The median age of the sample was 30 years (range $12-98$ years); 173 subjects (42\%) were female.

IDENTIFYING PATIENTS WITH ALCOHOL RELATED PROBLEMS

A total of 115 (28\%) patients were considered to have alcohol related problems on the basis of either the CAGE questionnaire or the staff assessment. Table 1 shows the distribution of presenting complaints. Alcohol was a significant contributing factor to head injuries and psychiatric presentations. There was a marked difference in the distribution of presenting complaints between patients with alcohol related problems and those without $\left(\chi^{2} 118.1\right.$, df 25, p<0.001).

Compared with other patients, these patients were younger (median age: $27 v 31$ years, $\mathrm{p}<0.02$, Mann-Whitney U test) and consumed more alcohol (median number of units consumed daily: $5 v 2, \mathrm{p}<0.001$, Mann Whitney $U$ test). Patients with alcohol related problems were more likely to present out of hours $\left(\chi^{2}\right.$ 13.05, df 5, p<0.05), but no more likely to present on particular days of the week. When asked whether they thought their attendance was alcohol related, 49 of 115 (43\%) patients with a presumed alcohol related attendance reported that it was, compared with 4 of 298 $(1.3 \%)$ of patients without a presumed alcohol related reason for attendance $\left(\mathrm{p}<0.01, \chi^{2}\right.$ test).

COMPARING THE CAGE QUESTIONNAIRE WITH STAFF ASSESSMENT

Thirty six patients were identified as problem drinkers on the basis of both their responses to the CAGE questionnaire and the staff assessment. Thirty nine patients were identified as problem drinkers on the basis of the CAGE only, and 40 patients were identified as problem drinkers on the basis of the staff assessment only. Comparing those identified by the CAGE with those identified by staff, the CAGE positive group were more likely to be male (33 of $39(85 \%)$ male $v 19$ of $40(48 \%)$ male, $\mathrm{p}<0.01, \chi^{2}$ test). There was no difference in age or number of units consumed or the day of presentation in the two groups. Figure 1 shows the time of presentation for alcohol related attendances to the ED. The CAGE positive group were more likely to present during normal working hours $\left(\chi^{2} 10.4\right.$, df 5, $\mathrm{p}=0.06)$. The CAGE positive subjects were more likely to acknowledge their attendance at the ED was attributable to alcohol (24 of 39 $(90 \%) v 4$ of $40(10 \%), \mathrm{p}<0.01, \chi^{2}$ test $)$.

\section{Discussion}

This study is one of the few UK studies to be conducted on patients who attend the ED department with alcohol related problems and the only one to our knowledge to use a representative flow sample. We were successfully able to screen 413 of 429 patients (96\%), a much higher proportion than reported in previous research. It confirms the findings of work from both the UK and USA that alcohol is a significant contributing factor to $\mathrm{ED}$ 
attendance. ${ }^{1-3} 1011$ Nearly one third of presentations to our ED were alcohol related to some degree.

We have shown that it is possible to detect large numbers of patients with alcohol problems in EDs using relatively unsophisticated screening tools, in this case a CAGE questionnaire or a staff assessment. In addition our results suggest that certain presenting complaints, such as head injuries or psychiatric presentations should alert ED staff to the possibility of alcohol misuse.

The absence of a gold standard for the detection of alcohol related psychiatric diagnoses was a potential weakness of this study. However, an in depth standardised interview is time consuming, must be administered by a trained interviewer, and is less likely to be acceptable to patients in the ED than the measures used here. Even screening tools may sometimes be impractical to administer. A study carried out in a London teaching hospital estimated that administering a two minute questionnaire to all adult patients in the ED would add 35 hours to each working week, and concluded that time pressures were a major factor in low pick up rates. ${ }^{11}$

Patients with alcohol problems who present to EDs can be helped. A recent uncontrolled UK study found that almost two thirds of patients referred to an Alcohol Health Worker based in the ED attended their appointment, and in those 71 patients successfully followed up at six months the mean reduction of alcohol intake was an impressive $43 \% .{ }^{14}$ However, this study was carried out using a highly selected sample. Other studies have reported a much less favourable outcome. For example a study of over 4000 patients attending an ED in the north of England suggested that only $41 \%$ of patients identified as drinking excessively could be offered help, and of these nearly $90 \%$ declined to be treated. ${ }^{12}$ Studies carried out in primary care have suggested that brief interventions are effective in reducing alcohol consumption ${ }^{15}$ but the results need to be interpreted cautiously because of drop out rates of up to $80 \% .{ }^{16}$ It is also unclear what the active ingredients of such treatments might be and who might most benefit from receiving them. ${ }^{17}$

This study suggests that patients with alcohol problems who present to the ED are not a homogenous group. Different screening methods identify different groups of patients, who in turn might respond to different forms of intervention.

The CAGE may identify cases of longer standing alcohol misuse, a group that research suggests might be more difficult to help. ${ }^{15}$ However, these patients seem to recognise they have an alcohol problem when they attend the $\mathrm{ED}$ and this is a good opportunity to intervene if the patient can be engaged immediately. This could be facilitated by an alcohol health worker based in the department, and is made more practical because CAGE positive patients attend during normal working hours.

Those patients identified on the basis of staff assessment are often intoxicated and lack insight into the nature of their problem when they attend the ED. Any immediate intervention at the time of presentation is unlikely to succeed and may be impractical to provide as these patients tend to attend out of hours. An alternative approach that may be of benefit is to give patients information cards inviting them back to the ED the next day to discuss their alcohol problem when they are no longer intoxicated. ${ }^{10}$ These people are hazardous drinkers and are perhaps less likely to be truly alcohol dependent than those patients identified by the CAGE. Evidence suggests that they are more likely to respond to brief interventions, ${ }^{15}$ perhaps delivered by non-specialist staff.

In conclusion, more patients attend EDs than are seen in hospital outpatient clinics. Presentation to the ED with alcohol related problems therefore represents a real opportunity to intervene. Patients could be picked up early in the natural history of their alcohol misuse when we know that intervention is more likely to succeed. We may also identify patients that are not currently known to health services. Effective treatments will prevent longer term social and health related difficulties. They may also reduce repeat attendance at the $\mathrm{ED}$, up to a fifth of which is attributable to alcohol misuse. ${ }^{18}$ The most basic strategies would include some form of screening not only to identify the extent of the problem, but (as happened in this study) to raise awareness within individual EDs. However, screening has the potential to hugely increase the workload of both EDs and community alcohol services. Potential solutions might include employing alcohol health workers in the $\mathrm{ED}^{14}$ or training non-specialist staff to provide brief interventions, but this may not be an option in many smaller or hard pressed departments. Alerting patients' general practitioners is also an important part of management especially if, as often happens, the patient is not amenable to advice at the time of presentation to the ED.

Although these general strategies may be helpful, it is unlikely that "blanket" approaches to intervention will be effective. This study and previous work ${ }^{17}$ suggest that patients presenting to the ED with alcohol problems are a complex and heterogeneous group. Further research examining the efficacy and feasibility of different alcohol treatment approaches is needed to enable us to target specific interventions to those patients who might most benefit.

We would like to thank all the patients and staff who participated in this study, S McManus for her helpful advice in setting up the study, and an anonymous referee for helpful comments on an earlier draft of this paper.

Funding: none.

Conflicts of interest: none.

\section{Appendix}

The questionnaire

(A) Health professional assessment

Do you think this patient's attendance is alcohol related?

(B) Patient assessment

Presenting complaint

How much do you drink?

Do you think your attendance is alcohol related? $C A G E$ questionnaire 
Have you ever felt you ought to cut down on your drinking?

Have people ever annoyed you by criticising your drinking?

Have you ever felt bad or guilty about drinking?

Have you ever had a drink in the morning to steady your nerves or get rid of a hangover?

1 Minugh PA, Nirenberg TD, Clifford PR, et al. Analysis of alcohol use clusters among subcritically injured emergency department patients. Acad Emerg Med 1997;4:1059-67.

2 Holt S, Stewart K, Dixon JM, et al. Alcohol and the emergency service patient. $B M \mathcal{F}$ 1980;281:631-40.

3 Silver BA, Sporty LD. Behavioral correlates and staff recognition of alcohol use in a university hospital trauma service. nition of alcohol use in a univer
Psychosomatics 1990;31:420-5.

4 Chang G, Astrachan BM. The emergency department surveillance of alcohol intoxication after motor vehicle accidents. $7 A M A$ 1988;260:2533-6.

5 Cherpitel CJ. Alcohol and violence related injuries: an emergency room study. Addiction 1993;88:79-88.

6 Sonderstrom C, Dischinger P, Smith G, et al. Psychoactive substance dependence among trauma center patients. JAMA 1992;267:2756-9.

7 Clifford PR, Sparadeo F, Minugh PA, et al. Identification of hazradous/harmful drinking among subcritically injured patients. Acad Emerg Med 1996;3:239-45.

8 Davidson P, Koziol-McLain J, Harrison L, et al. Intoxicated ED patients: a 5-year follow-up of morbidity and mortality. Ann Emerg Med 1997;30:593-7.
9 Lowenstein SR, Weissberg MP, Temy D. Alcohol intoxication, injuries, and dangerous behaviors and the revolving emergency department door. F Trauma 1990;30:1252-8.

10 Green M, Setchell J, Hames P, et al. Management of alcohol abusing patients in accident and emergency departments. $\mathcal{F}$ $R$ Soc Med 1993;86:393-5.

11 Smith SGT, Touquet R, Wright S, et al. Detection of alcohol misusing patients in accident and emergency departments: the Paddington alcohol test (PAT) $\mathcal{F}$ Accid Emerg Med 1996;13:308-12.

12 Peters J, Brooker C, McCabe C, Short N. Problems encountered with opportunistic screening for alcohol related problems in patients attending an accident and emergency department. Addiction 1998;93:589-94.

13 Ewing JA. Detecting alcoholism: the CAGE questionnaire. FAMA 1984;252:1905-7.

14 Wright S, Moran L, Meyrick M, et al. Intervention by an Alcohol Health Worker in an accident and emergency department. Alcohol Alcohol 1998;33:651-6.

15 Bien TH, Miller WR, Tonigan JS. Brief interventions for alcohol problems: a review. Addiction 1993;88:315-35.

16 Edwards AG, Rollnick S. Outcome studies of brief alcohol intervention in general practice: the problem of lost intervention in general practice: the
subjects. Addiction 1997;92:1699-704.

17 Drummond DC. Alcohol interventions: do the best things come in small packages? Addiction 1997;92:375-9.

18 Andren KG, Rosenqvist U. Heavy users of an emergency department: psychosocial and medical characteristics, other health care contacts and the effect of a hospital social worker intervention. Soc Sci Med 1985;21:761-70. 